\title{
Author's reply to the commentary by Gilpin et al.
}

M. Martini * (1,2), D. Perez-Marcos (1,2) and M.V. Sanchez-Vives $(1,2,3,4)$

(1) Institut d'Investigacions Biomèdiques August Pi i Sunyer (IDIBAPS), Barcelona, Spain

(2) EVENT-Lab, Facultat de Psicologia, Universitat de Barcelona, Barcelona, Spain

(3) Institució Catalana Recerca i Estudis Avançats (ICREA), Barcelona, Spain

(4) Departamento de Psicología Básica, Universitat de Barcelona, Barcelona, Spain

Corresponding author:

Matteo Martini, PhD

EventLab

Universitat de Barcelona

Facultat de Psicologia,

Departament de Personalitat, Avaluació i Tractaments Psicològics,

Campus de Mundet - Edifici Teatre,

Passeig de la Vall d'Hebron 171,

08035 Barcelona (Spain)

E-mail: mmartini@clinic.ub.es 
We thank the authors of the commentary entitled, 'Exploring the role of body ownership, vision and virtual reality on heat pain threshold' (Gilpin et al., 2014) focused upon our recent paper, 'Modulation of pain threshold by virtual body ownership' (Martini et al., 2014), which raised interesting issues worth discussing. In our study, we described how the vision of a virtual arm over which we had induced ownership has analgesic effects, similarly to the reported effect of seeing one's real arm (Longo et al., 2009, 2012). In their comments, Gilpin et al. raised questions about mechanisms behind the analgesia observed inside virtual environments. They considered the role of virtual environments per se as inductor of analgesia, based upon the fact that distraction while in an immersive virtual reality can in itself decrease pain during painful medical procedures (e.g., Hoffman et al., 2011). Hoffman et al., however, used virtual environments to decrease pain based upon distraction, by creating highly engaging and interactive environments where patients played a game shooting snowballs while undergoing painful medical procedures concerned with treatment of burns. Participants had no virtual body and could not see their real body that was obscured by the head-mounted display. In our experiments, there were no distractions and participants were simply looking down at their virtual body. Accumulated research in virtual reality provides no reason to believe that virtual environments are analgesic per se. Indeed, in our study (Martini et al., 2014), we demonstrate that the pain threshold is significantly higher in the synchronous condition where ownership of the virtual arm had been induced than in the condition where instead of an arm, there is a cylinder on the table, even when both conditions occurred in a virtual environment. Another issue raised by Gilpin et al. is the difficulty of differentiating the role of 'vision of the arm' and 'body ownership' on the analgesia effect that we found, stating that our study design does not allow disentanglement of the relative contribution of these two factors. To support this claim, they argue that in our virtual synchronous condition, as opposed to the control outside virtual reality, the role of vision could not be dissociated from the effects of merely being inside the virtual environment. They argue that 'the virtual synchronous condition led to higher pain thresholds than viewing a virtual object, yet vision cannot be disentangled from the effects of ownership, as 
confirmed by the much lower ratings of ownership in the virtual object condition'. We think, instead, that our results and experimental design did allow us to disentangle the influence of vision, virtual reality and body ownership on pain perception. As mentioned above, we demonstrated that there is no difference in pain threshold between being outside or inside the virtual environment. Further, the fact that the synchronous and not the asynchronous condition induces a significantly higher pain threshold than the control conditions demonstrates that simply the vision of the virtual body is not sufficient to induce changes in pain perception, but ownership of that body is required. Gilpin et al. mentioned the work by Mohan et al. (2012) to state that the rubber hand illusion (RHI) has no effect on experimental pain levels in the real limb, as shown by two independent experiments which the authors say are 'well-powered'. However, it should be noted that Mohan et al.'s experiments are based upon 16 (experiment 1) and 20 (experiment 2) subjects, while a similar work conducted by Hegedus et al. based upon 30 subjects found that the RHI does have an effect on increasing pain threshold as compared to the asynchronous condition (Hegedüs et al., 2014). In our study (Martini et al., 2014) where we induced the virtual hand illusion (VHI; Slater et al., 2008; Sanchez-Vives et al., 2010) instead of the RHI, we obtained data from 24 subjects and we also did not find a significant difference in pain threshold between the synchronous and asynchronous conditions, which would be indeed in agreement with Mohan et al. Nevertheless, our findings revealed that with respect to the vision of real or virtual objects, the vision of a virtual body part, which was felt as owned, did have analgesic effect. This is in line with Longo's findings that the vision of one's own real limb is analgesic, as compared to the vision of an object or also compared to the vision of somebody else's limb (Longo et al., 2009). So the critical aspect appears to be not simply seeing a body part, but that there needs to be an illusion of ownership over that body part. Given the well-known modulatory effect that attention has on pain, we cannot rule out that the virtual synchronous condition could have been more distracting than the other conditions by activating multisensory networks, as suggested by Gilpin et al. However, although the synchronous 
and the asynchronous conditions showed very similar levels of attention, only the synchronous condition resulted in a significant increase of pain threshold compared to control conditions in our experiment. Furthermore, it has been recently argued that the body-related visual analgesia would not merely rely upon distraction but rather on a complex reorganization of the receptive fields of the neurons within the primary somatosensory cortex (Haggard et al., 2013).

Conflict of interest: none declared.

\section{References}

Gilpin, H.R., Bellan, V., Gallace, A., Moseley, G.L. (2014). Exploring the roles of body ownership, vision and virtual reality on heat pain threshold. Eur J Pain 18, 900-901.

Haggard, P., lannetti, G.D., Longo, M.R. (2013). Spatial sensory organization and body representation in pain perception. Curr Biol 23, R164-R176.

Hegedüs, G., Darnai, G., Szolcsányi, T., Feldmann, A., Janszky, J., Kállai, J. (2014). The rubber hand illusion increases heat pain threshold. Eur J Pain 18, 1173-1181.

Hoffman, H.G., Chambers, G.T., Meyer, W.J. 3rd, Arceneaux, L.L., Russel, W.J. , Seibel, E.J., Richards, T.L., Sharar, S.R., Patterson, D.R. (2011).

Virtual reality as an adjunctive non-pharmacologic analgesic for acute burn pain during medical procedures. Ann Behav Med 41, 183-191.

Longo, M.R., Betti, V., Aglioti, S.M., Haggard, P. (2009). Visually induced analgesia: Seeing the body reduces pain. J Neurosci 29, 12125-12130.

Longo, M.R., lannetti, G.D., Mancini, F., Driver, J., Haggard, P. (2012). Linking pain and the body: Neural correlates of visually induced analgesia. J Neurosci 32, 2601-2607. 
Martini, M., Perez-Marcos, D., Sanchez-Vives, M.V. (2014). Modulation of pain threshold by virtual body ownership. Eur J Pain 18, 1040-1048.

Mohan, R., Jensen, K.B., Petkova, V.I., Dey, A., Barnsley, N., Ingvar, M., McAuley, J.H., Moseley, G.L., Ehrsson, H.H. (2012). No pain relief with the rubber hand illusion. PLoS ONE 7, e52400.

Sanchez-Vives, M.V., Spanlang, B., Frisoli, A., Bergamasco, M., Slater, M. (2010). Virtual hand illusion induced by visuomotor correlations. PLoSONE 5, e10381.

Slater, M., Perez-Marcos, D., Ehrsson, H.H., Sanchez-Vives, M.V. (2008). Towards a digital body: The virtual arm illusion. Front Hum Neurosci 2, 6. 